\title{
A Multilead Fusion Based QRS Complex Detection Method on 12-lead Electrocardiogram Signals
}

\author{
Wei Zhao ${ }^{1}$, Yanwu Xu ${ }^{1}$, Cong Yan $^{1}$, Jing $\mathrm{Hu}^{1}$, Dongya $\mathrm{Jia}^{1}, \mathrm{Xu} \mathrm{Sun}^{1}$, Tianyuan $\mathrm{You}^{1}$ \\ ${ }^{1}$ Central Research Institute for Guangzhou Shiyuan Electronics Co., Ltd, Guangzhou, China
}

\begin{abstract}
The electrocardiogram (ECG) signal records the electrical activity of the heartbeat on one or more leads. The QRS complex is thought to be one of the most prominent ECG waves due to its large slope and high amplitude, and the accurate detection of the QRS complex is an essential function for the ECG analysis algorithms. Although numerous QRS complex detectors have been proposed, few method payed attention to analyzing the information simultaneous present in multilead ECG signals, which would be resistant to the noise. In this work, we presented a multilead QRS complex detection approach on 12-lead ECG signals. After detecting the QRS complex on each single lead, a novel multilead fusion strategy was applied to cluster the QRS complexes. The proposed method was evaluated on the St.Petersburg Institute of Cardiological Technics 12-lead Arrhythmia Database. The detection error rate (DER) of QRS complex ranged from $1.17 \%$ to $13.78 \%$ on each single lead. After the multilead fusion, the DER was reduced to $0.39 \%$. Experimental results show that the proposed method would be useful to produce an accurate $Q R S$ complex detection result.
\end{abstract}

\section{Introduction}

The electrocardiogram (ECG) is an kind of electrical biosignal recording the electrical activity of the heartbeat over a period of time on one or more leads. The QRS complex which corresponds to the activity of ventricular depolarization, is thought to be the most prominent wave due to its large slope and high amplitude. The accurate detection of the QRS complex is an essential step in the automatical ECG analysis algorithm, which can be used to measure the heart rate, determine the rhythm of heartbeat and provide the fiducial points to detect other waves like $\mathrm{P}$ and $\mathrm{T}$ wave[1].

Several approaches have been proposed to detect the QRS complex since 1980s. In the time domain, the slope of $\mathrm{R}$ wave was a popular feature used to locate the position of the QRS complex. Pan and Tompkins [2] identi- fied the QRS complex by thresholding the auxiliary signal, which was calculated according to the slope and width of the ECG waves. The wavelet is another widely used type of tool for the detection of the QRS complex. For example, the work [3] applied the Haar wavelet transform by thresholding the productions of the detail coefficients of $t$ wo levels. In order to benefit from both the wavelet and time domain analysis, Fernandez et.al [4] combined the quadratic spline wavelet with the Pan and Tompkins' detector to detect the QRS peaks.

Besides, the Hilbert transform [5], morphological transform [6], optimized knowledge-based [7], and machine learning based techniques such as support vector machine [8], neural network [9] and K-Means [10] were also applied to develop the QRS complex detectors.

Considering that the twelve-lead circuitry was used for the signal acquisition in electrocardiograph, and the multilead analysis of QRS complex would be resistant to presence of noise from the sources like electrode motion and electromyographic (EMG), some researchers tried to detect QRS complex on two or more leads. The work [11] estimated a confidence value for each QRS complex candidate according to the RR intervals. The work [12] applied the vote based fusion strategy for the multilead fusion after detecting QRS complex on 12 single lead. This method achieved a high precision, but relatively lower sensitivity.

In this work, we designed a novel QRS complex detection method on multilead signals. The St.Petersburg Institute of Cardiological Technics 12-lead Arrhythmia Database [13] was adopted to evaluate the proposed method. Experimental shows that the proposed multilead fusion strategy was advantageous to improve the performance of QRS complex detection, and our method can accurately estimate the position of QRS complex on multilead signals.

\section{Proposed Method}

The flowchart of the proposed multilead QRS complex detection method was shown in Fig. 1. At first, the QRS detector was adopted to detect the QRS complex on the single lead $\mathrm{ECG}_{i}, i=1,2, \ldots, n$. Then the multilead fu- 


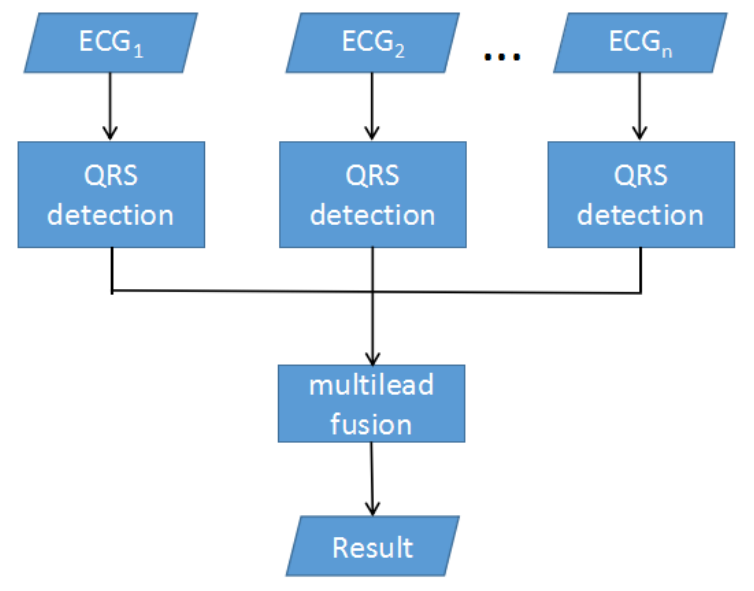

Figure 1. Flowchart of the proposed multilead QRS complex detection method

sion strategy was applied to cluster the positions of QRS complex on different leads and identify the clustered QRS complex candidates were true or not. Finally the average positions of true QRS complexes belonging to the same heartbeat were output.

\subsection{QRS complex detection}

The well-known PT detector [2] was adopted to detect the QRS complex on each single lead. Firstly, the ECG signal was filtered by a cascade of a lower-pass filter and a high-pass filter. The difference functions of two filters was given in Eq. 1 and Eq. 2 respectively, where the $x_{i}$ and $y_{i}$ were the $i-t h$ sample of the input and output data respectively.

$$
\begin{gathered}
y_{i}=2 y_{i-1}-y_{i-2}+x_{i}-2 x_{i-6}+x_{i-12} \\
y_{i}=32 x_{i-16}-\left[y_{i-1}+x_{i}-x_{i-32}\right]
\end{gathered}
$$

Then the derivative of filtered signals was calculated by a five-point difference function given in Eq.3.

$$
y_{i}=(1 / 8) \cdot\left[-x_{i-2}-2 x_{i-1}+2 x_{i+1}+x_{i+2}\right]
$$

In the next step, the derivative signal was squared point by point. Finally, the moving-window integration of the squared signal was calculated and used as the auxiliary signal. Eq.4 gives the integrated function, where the $l$ is the width of the moving-window (150ms was suggested by [2]).

$$
y_{i}=(1 / l) \cdot\left[x_{i-l+1}+x_{i-l+2} \ldots+x_{i}\right]
$$

The QRS complex corresponds to the rising edge of the auxiliary signal. So it can be identified by thresholding the amplitude of the auxiliary signal. If there was no QRS complex detected during a specific interval, the process of searchback was performed and the maximal peak between the two given thresholds during the interval was treated as the QRS complex. Furthermore, the neighboring peaks whose interval was smaller than $0.36 \mathrm{~s}$ was checked in order to remove the high $\mathrm{T}$ wave which would be misclassified as the QRS complex. The details of this QRS complex detector can be referred to the paper [2].

\subsection{Multilead fusion}

After detecting the QRS complex on each single lead, the following strategy was used to fusion the QRS complexes:

1. A fusion window with a width of $200 \mathrm{~ms}$ was opened by the first detected QRS complex.

2. The QRS complexes within the fusion window were treated as the same QRS complex.

3. The first QRS complex out of the current fusion window was treated as a new QRS complex, and opened a new fusion window.

4. Repeated the step 2, until all QRS complex were assigned.

Although the QRS complex detector may produce some false positives (falsely detected QRS complex) or false negatives (missed QRS complex) on the single lead, this problem can be solved by the vote strategy given in Eq.5. Define $d_{i}=1$ if the QRS complex was detected on the lead $_{i}$, and $d_{i}=0$ if the QRS complex was not detected on the lead ${ }_{i}$. If the sum of $d_{i}, i=1, \ldots, n$ was smaller than the threshold, for example, the $n / 2$ which means that the QRS complex was detected on smaller than half of the number of leads, this QRS complex was thought to be the false positive. Otherwise, it was thought as the true positive.

$$
\text { QRS complex }= \begin{cases}\text { true positive, } & \sum d_{i} \geq n / 2 \\ \text { false positive, } & \text { otherwise }\end{cases}
$$

\section{Experimental Results}

The proposed method was implemented in Python 3.6 and evaluated on the St.Petersburg Institute of Cardiological Technics 12-lead Arrhythmia Database [13]. This database contains 75 12-lead ECG recordings. These records are 30 minutes long, sampled at $257 \mathrm{~Hz}$. There are over 175000 beat annotation in the reference annotation files.

The performance of the QRS complex detection and multilead fusion was measured by sensitivity (Se), precision $(+\mathrm{P})$ and detection error rate (DER) given in Eq.6 . If the margin between a detected QRS complex and annotated beat was smaller than $150 \mathrm{~ms}$, which was suggested by the standard of ANSI/AAMI EC57, this QRS complex was treated as the TP (true positive). Otherwise, it was treated 
Table 1. Results of combinations of QRS complex detection and multilead fusion strategy

\begin{tabular}{ccccc}
\hline Detection & Fusion & $\mathrm{Se}(\%)$ & $+\mathrm{P}(\%)$ & $\mathrm{DER}(\%)$ \\
\hline w searchback & one window & 99.63 & 99.97 & 0.39 \\
w searchback & two windows & 93.19 & 99.98 & 6.83 \\
wo searchback & one window & 99.43 & 99.97 & 0.59 \\
wo searchback & two windows & 93.75 & 99.98 & 6.27 \\
\hline
\end{tabular}

as the FP (false positive). The undetected annotated beat was thought as the FN (false negative).

$$
\begin{aligned}
S e & =\frac{\mathrm{TP}}{\mathrm{TP}+\mathrm{FN}} \\
+P & =\frac{\mathrm{TP}}{\mathrm{TP}+\mathrm{FP}} \\
D E R & =\frac{\mathrm{FP}+\mathrm{FN}}{\mathrm{TP}+\mathrm{FN}}
\end{aligned}
$$

The work [12] was adopted to compared with the proposed method. This work consists of two stages: detection of QRS complex and multilead fusion of the QRS complexes. In the stage of QRS complex detection, the PT detector was also adopted to detect the QRS complex, but the step of the searchback for the missed QRS complex was not performed in order to reduce the delay of detection. In the stage of the multilead fusion, two time windows were applied: the fusion window of $100 \mathrm{~ms}$ and blind window of $250 \mathrm{~ms}$. The first detected QRS complex opened the fusion window, and the QRS complex candidates within this window were thought as the same QRS complex and the QRS complex candidates within the blind-eye window were ignored. The vote fusion strategy was used to identify the QRS complex candidates in the fusion window was true QRS complex or not.

The table 1 summarizes the performance of the different combinations of QRS complex detection and multilead fusion strategies. The proposed method achieved the lowest DER $(0.39 \%)$ and highest sensitivity $(99.63 \%)$ between the four methods. When the searchback was not performed, the DER and sensitivity of proposed one window based fusion strategy slightly changed to $0.59 \%$ and $99.43 \%$ respectively. For the two window based fusion strategy, when the searchback was and was not applied, the DERs were $6.83 \%$ and $6.27 \%$ respectively. The result of QRS complex detection on each single lead with and without searchback are given in table 2 and 3. Both two types of method achieved the best DER on the lead 7-th.

\section{Discussion}

Experimental results show the application of the searchback in the QRS detection can slightly improve the sensitivity and DER, and the proposed one time window based
Table 2. Results of QRS detection with searchback on each single lead

\begin{tabular}{cccc}
\hline ECG Lead & $\mathrm{Se}(\%)$ & $+\mathrm{P}(\%)$ & DER $(\%)$ \\
\hline 1 & 91.1 & 95.81 & 12.88 \\
2 & 95.6 & 99.06 & 5.3 \\
3 & 96.37 & 99.00 & 4.6 \\
4 & 98.95 & 99.17 & 1.88 \\
5 & 89.37 & 96.6 & 13.78 \\
6 & 93.96 & 99.33 & 6.68 \\
7 & 99.16 & 99.67 & 1.17 \\
8 & 98.95 & 99.73 & 1.32 \\
9 & 97.54 & 99.11 & 3.34 \\
10 & 96.47 & 98.82 & 4.69 \\
11 & 97.67 & 99.91 & 2.42 \\
12 & 97.42 & 99.38 & 3.19 \\
\hline
\end{tabular}

Table 3. Results of QRS detection without searchback on each single lead

\begin{tabular}{cccc}
\hline ECG Lead & $\mathrm{Se}(\%)$ & $+\mathrm{P}(\%)$ & $\mathrm{DER}(\%)$ \\
\hline 1 & 88.87 & 96.54 & 14.42 \\
2 & 94.54 & 99.22 & 6.2 \\
3 & 94.54 & 99.15 & 6.27 \\
4 & 97.44 & 99.32 & 3.22 \\
5 & 85.62 & 97.83 & 16.28 \\
6 & 92.33 & 99.53 & 8.1 \\
7 & 97.89 & 99.84 & 2.27 \\
8 & 97.42 & 99.74 & 2.83 \\
9 & 95.97 & 99.23 & 4.77 \\
10 & 94.92 & 98.97 & 6.07 \\
11 & 96.31 & 99.92 & 3.78 \\
12 & 96.52 & 99.48 & 3.98 \\
\hline
\end{tabular}

fusion strategy achieved significantly better performance than the fusion strategy of two time windows (one fusion window and one blind-eye window).

In the stage of QRS detection, the PT detector identified the QRS complex by thresholding the auxiliary signal, and the value of threshold was adaptively adjusting according to the amplitude of the auxiliary signals. Because the presence of the ventricular ectopic beat with a high amplitude and large slope would lead to a high threshold, the process of the searchback was useful to locate the missed QRS complex although the searchback would produce some false positives. Table 2 and 3 show that the introduction of the searchback can increase the sensitivity while slightly reduce the precision of the QRS complex detection on all single leads.

In the stage of multilead fusion, the goal of the fusion strategy was to remove the false positives and detect the false negatives. In the work [12], only the QRS complexes candidates within the fusion window were be checked by the vote strategy, and candidates within the subsequence 
blind-eye window were ignored. It is advantageous to remove the false positives like $\mathrm{T}$ waves. So this two time windows based fusion strategy achieved the highest precision of $99.98 \%$. However, when the fusion window was opened by the false positives, the QRS complexes located within the blind-eye window were ignored. Therefore sensitivity of the QRS detection was effected. In our experiments, the sensitivity of the two fusion window strategy was $93.19 \%$ and $93.75 \%$ when the searchback was performed and not performed respectively.

In order to improve the performance of the multilead fusion, considering that most of the false positives were detected on few lead, in this work the QRS complex candidates detected on single leads were analyzed by only one time window, and the blind window was not applied to remove the false positives. Although the precision of proposed strategy was slightly lower (99.97\% vs. 99.98\%), the sensitivity and DER were significantly better than two fusion windows strategy. When the searchback was performed, the sensitivity and DER for the proposed fusion strategy were $99.63 \%$ and $0.39 \%$ respectively, which were $93.19 \%$ and $6.83 \%$ for the two fusion windows strategy. When the searchback was not performed, the proposed fusion strategy achieved the sensitivity of $99.63 \%$ and DER of $0.39 \%$, while result of the two fusion windows strategy were $93.19 \%$ and $6.83 \%$ respectively.

\section{Conclusion}

In this paper, we developed a novel method to detect QRS complex on multilead ECG signals. After detecting the QRS complex on each single lead by using the famous PT detector, a short window was used for the multilead fusion, and the vote strategy was employed to remove the false positives. If the QRS complex was detected on equal or more than half of the number of leads, it was treated as the true positive. Experimental results show that the proposed method can accurately detect the QRS complex on the 12-lead ECG recordings. In future works, we will try to adopt sophisticated fusion strategy and evaluate the performance of proposed method on more databases.

\section{References}

[1] Kohler BU, Hennig C, Orglmeister R. The principles of software qrs detection. IEEE Engineering in Medicine and Biology Magazine Jan 2002;21(1):42-57. ISSN 07395175.

[2] Pan J, Tompkins WJ. A real-time qrs detection algorithm. IEEE Transactions on Biomedical Engineering March 1985;BME-32(3):230-236. ISSN 0018-9294.

[3] Zidelmal Z, Amirou A, Adnane M, Belouchrani A. Qrs detection based on wavelet coefficients. Comput Methods Prog Biomed September 2012;107(3):490-496. ISSN 0169-2607.
[4] Fernandez J, Harris M, Meyer C. Combining algorithms in automatic detection of r-peaks in ecg signals. In 18th IEEE Symposium on Computer-Based Medical Systems (CBMS'05). ISSN 1063-7125, June 2005; 297-302.

[5] Arzeno NM, Deng ZD, Poon CS. Analysis of firstderivative based qrs detection algorithms. IEEE Transactions on Biomedical Engineering Feb 2008;55(2):478-484. ISSN 0018-9294.

[6] Sun Y, Chan KL, Krishnan SM. Characteristic wave detection in ecg signal using morphological transform. BMC Cardiovascular Disorders Sep 2005;5(1):28. ISSN 14712261.

[7] Elgendi M. Fast qrs detection with an optimized knowledge-based method: Evaluation on 11 standard ecg databases. PLOS ONE 09 2013;8(9):1-18.

[8] qiang Zeng Z, Wu Q, Wu KS. Simplified support vector machine method for qrs wave detection. In 2009 IEEE 10th International Conference on Computer-Aided Industrial Design Conceptual Design. Nov 2009; 1427-1429.

[9] Shuyan W. Automatic detection of qrs complexes using quantum neural networks. In 2008 International Conference on BioMedical Engineering and Informatics, volume 2. ISSN 1948-2914, May 2008; 306-309.

[10] Merino M, Gomez IM, Molina AJ. Envelopment filter and $\mathrm{k}$-means for the detection of qrs waveforms in electrocardiogram. Medical Engineering and Physics 2015;37(6):605 - 609. ISSN 1350-4533.

[11] Torbey S, Akl SG, Redfearn DP. Multi-lead qrs detection using window pairs. In 2012 Annual International Conference of the IEEE Engineering in Medicine and Biology Society. ISSN 1094-687X, Aug 2012; 3143-3146.

[12] A.Ledezma C, Perpian G, Severeyn E, Altuve M. Data fusion for qrs complex detection in multi-lead electrocardiogram recordings. In 11 th International Symposium on Medical Information Processing and Analysis, volume 9681. 2015; $9681-9681-9$.

[13] Goldberger AL, Amaral LAN, Glass L, Hausdorff JM, Ivanov PC, Mark RG, Mietus JE, Moody GB, Peng CK, Stanley HE. PhysioBank, PhysioToolkit, and PhysioNet: Components of a new research resource for complex physiologic signals. Circulation 2000 (June 13);101(23):e215-e220. Circulation Electronic Pages: http://circ.ahajournals.org/content/101/23/e215.full PMID:1085218; doi: 10.1161/01.CIR.101.23.e215.

Address for correspondence:

Name: Wei Zhao, Yanwu Xu

Full postal address: No. 6, Yunpu 4th Rd., Huangpu District, Guangzhou, China (510530)

E-mail address: zhaowei@cvte.com,xuyanwu@cvte.com 